www.jmscr.igmpublication.org

Impact Factor 5.84

Index Copernicus Value: 83.27

ISSN (e)-2347-176x ISSN (p) 2455-0450

crossref DOI: _https://dx.doi.org/10.18535/jmscr/v5i3.81

Journal Of Medical Science And Clinical Research

\title{
Microbiological Significance in Catheter Associated Urinary Tract Infections of Traumatic Spinal Cord Injury Patients
}

\author{
Authors \\ Mohan Raj Manjalavil ${ }^{1}$, Vasudevan T.K ${ }^{2}$, Sooraj Rajagopal ${ }^{3}$, \\ Sreedevi Menon Parappil ${ }^{4}$ \\ ${ }^{1}$ Associate Professor, Dept. of Physical Medicine \& Rehabilitation, Govt. Medical College, Kozhikode. \\ ${ }^{2}$ Professor, Dept. of Physical Medicine \& Rehabilitation, Govt. Medical College, Kottayam. \\ ${ }^{3}$ Associate Professor, Dept. of Physical Medicine \& Rehabilitation, Govt. Medical College, Kozhikode \\ ${ }^{4}$ Professor, Dept. of Physical Medicine \& Rehabilitation, Govt. Medical College, Kozhikode \\ Corresponding Author \\ Dr Mohan Raj Manjalavil \\ Associate Professor Dept. of Physical Medicine \& Rehabilitation, Govt. Medical College, Kozhikode \\ Kerala. India 673008. \\ Email: rajmanjalavil@yahoo.co.in Mob: 9447478276
}

\begin{abstract}
Background: Symptomatic urinary tract infections are one of the common causes of morbidity in spinal cord injury patients. In hospital acquired infections, bacteria reach the bladder either through urine present in the tube or from the extension of bowel flora. The organisms like Klebsiella, Pseudomonas, etc assume increasing importance apart from E. coli.
\end{abstract}

Objectives

1. To study the prevalence of micro pathogens like Klebsiella, Pseudomonas, Proteus etc. in catheter associated urinary tract infections.

2. To study sensitivity of micro pathogens to the antibiotics in patients with spinal cord injury.

Materials And Methods: 100 patients with traumatic spinal cord injury are selected for this study. Blood routine, urine routine, blood urea, serum creatinine, blood sugar (fasting \& postprandial) and ultrasound Kidney Ureter Bladder are done to assess the present condition and detect any complications. Urine culture and sensitivity tests are also performed and the results are analysed.

Setting and design: Cross sectional study in a tertiary level multispecialty hospital.

Results: The main age group affected are between 31- 40 yrs. The commonest organism seen in urine culture and sensitivity are E. coli (42\%), Klebsiella (28\%), Pseudomonas (16\%), Staphylococcus (8\%), Enterococcus (6\%) etc. There is increase in resistance to the drugs like ampicillin, ciprofloxacin and norfloxacin.

Conclusion: It was found that, in catheter associated UTIs the prevalence of Klebsiella, Pseudomonas and staphylococcus are much more than in community acquired UTIs. Multiple drug resistance are also found to be increased.

Keywords: Spinal cord injury, Catheter, Urinary Tract Infections, Culture and sensitivity, Antibiotics. 


\section{INTRODUCTION}

Urinary tract is considered as a single anatomic unit extending from kidney to the urethra. Microorganisms enter the urinary tract mainly through the urethra. Epidemiologically, urinary tract infections (UTIs) are of 2 types - catheter associated (nosocomial) and noncatheter associated (community acquired). Majority of urinary tract infections in spinal cord injury patients are associated with catheters and other urine collection devices. The diagnosis of UTIs in these patients is not easy, as the bladder sensation is affected.

According to the Kass concept, significant bacteriuria is seen when the count of $\geq 10^{5}$ colony forming units $(\mathrm{CFU}) / \mathrm{ml}$ of urine. Low count of < $10^{4} \mathrm{CFU} / \mathrm{ml}$ is considered as insignificant indicates presence of commensal bacteria ${ }^{1}$. However urine specimens obtained by suprapubic aspiration or catheterization and in samples from a patient with an indwelling catheter, colony counts of $10^{2}-10^{4} / \mathrm{mL}$ generally indicate infection ${ }^{2}$.

True UTI should be differentiated from bacterial colonisation (asymptomatic bacteriuria). In spinal cord injury, bacteriuria is common which represents only colonisation, not UTI. True UTI in SCI should meet 3 criteria. These are 1) presence of bacteria in urine 2) an increase in white blood cells in urine (pyuria) and 3) the onset of new symptoms. The new onset signs and symptoms are fever, urinary incontinence, heamturia, malaise, lethargy, cloudy and foul smelling urine, presence of pyuria, dysuria, increased spasticity, and autonomic dysreflexia.

Unnecessary antibiotic usage will lead to the development of drug resistance. However the prevalence of both asymptomatic bacteriuria and significant UTI are more in spinal cord injury patients $^{3}$.

Urine for the culture and sensitivity can be collected by the following methods - Midstream urine, catheter sample urine after cleaning the catheter with alcohol and suprapubic aspirate. If $\geq$ 3 types of organisms are grown, these are considered as contaminants, which may have been introduced into the urine sample from the perineal region, skin or external urethral meatus due to improper collection.

The common etiological agents of the urinary tract infection are mainly bacteria followed by virus, fungi and parasites. In patients without catheters, $80 \%$ of the acute infections are caused by gram negative bacilli like E. coli. In patients with catheters Klebsiella, Pseudomonas, Proteus, and Enterobacter plays a major role. Gram positive cocci like staphylococcus aureus and enterococci are rarely isolated from culture and sensitivity tests. Among the virus, adenovirus is the commonest. Candida albicans is the commonest fungi seen in diabetic patients. Neisseria gonorrhoeae, herpes simplex virus Trichimonas vaginalis are also seen in $\mathrm{UTI}^{4}$.

According to Burgdörfer et.al, antibiotic treatment should be started on the basis of microbiologic sensitivity testing ${ }^{5}$. Even though there is no consensus regarding the duration of treatment of UTIs, Everaert $\mathrm{K}$ et.al suggest that antibiotics should be given for a minimum period of 14 days in patients with UTI and fever ${ }^{6}$.

In this tertiary care multispecialty hospital, patients with spinal cord injury are admitted after 3 months of the accident for rehabilitation. Before admission majority of the patients are having indwelling catheters, which they have been changing every $3-4$ weeks. Most of the patients are having history of one or more symptomatic urinary tract infections, which are treated by antibiotics.

After admission to the department, all patients are investigated with urine $\mathrm{R} / \mathrm{E}$, blood $\mathrm{R} / \mathrm{E}$, blood urea, serum creatinine and USG KUB (Kidney Ureter Bladder). If the patient is having true symptomatic UTI, appropriate antibiotics depending on the culture and sensitivity will be prescribed, for a period of $7-14$ days.

\section{AIMS AND OBJECTIVES}

To study the prevalence of micro pathogens like Klebsiella, Pseudomonas, and Proteus in catheter associated urinary tract infections and its 
sensitivity to the antibiotics in patients with spinal cord injury who are having indwelling catheter. Associated demographic factors are also studied.

\section{MATERIALS AND METHODS Settings}

Tertiary care multi speciality hospital.

\section{Study Design}

Cross sectional study over a period of 12 months.

\section{Inclusion Criteria}

1. Spinal cord injury patients having features of true symptomatic UTI.

2. Patients between 3 months and one year.

3. Age between 20 to 60 years

4. Both sexes.

\section{Exclusion Criteria}

1. Patients with diabetes mellitus.

2. Patients with renal and vesical calculus.

3. Patients with renal disease.

4. Patients with urologic abnormalities.

\section{Study Tools}

1. Urine and blood R/E.

2. Renal function tests.

3. Urine culture and sensitivity tests.

4. Ultra sound KUB.

\section{Statistical Analysis}

Results were analysed using SPSS 16 version.

\section{Method}

100 patients who gave valid written consent and satisfying the inclusion criteria were selected for this study. Most of the patients are having indwelling catheters and a history of one or more UTIs before the admission. After thorough physical examination, routine blood and urine examination are done to assess the features of urinary infection. Renal function tests were done to assess the renal status. A fasting and postprandial blood sugar examination was also done to assess the diabetic status. This is followed by Ultra sound KUB to detect any structural or functional changes of the urinary tract. Finally, urine culture and sensitivity examinations were done to assess the type of pathogenic organisms and its sensitivity to antibiotics.

\section{RESULTS}

\section{Age distribution}

As displayed in Table 1, the main age group affected is between $31-40$ years $(36 \%)$.

Table No. 1: Distribution according to age

\begin{tabular}{|l|c|}
\hline Age & Percentage \\
\hline $21-30$ & 26 \\
\hline $31-40$ & 36 \\
\hline $41-50$ & 22 \\
\hline $51-60$ & 16 \\
\hline
\end{tabular}

\section{Sex distribution}

In the Pie chart No - 1, males are more affected than female. The major cause of spinal cord injury is fall from the trees.

Pie chart No 1: Distribution of gender

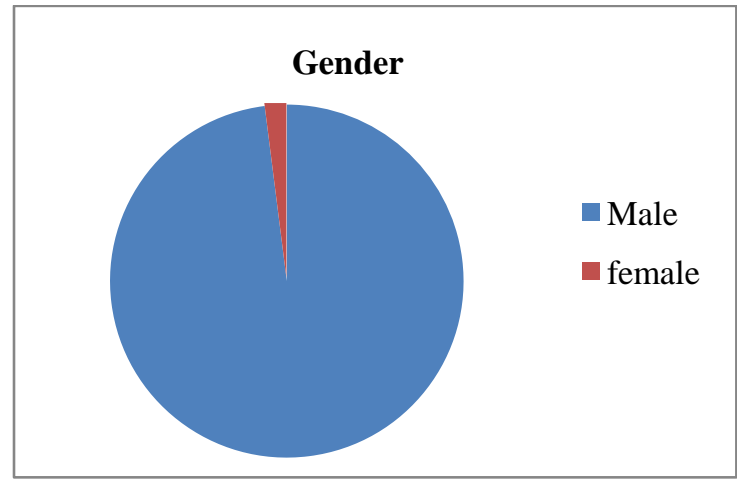

\section{Urine culture and sensitivity}

Graph - 1 showed that, the main organism seen in catheter associated UTIs is E. coli (42\%). This is followed by Klebsiella (26\%), Pseudomonas $(16 \%)$, Staphylococcus aureus $(8 \%)$, Proteus $(2 \%)$ and enterococci $(6 \%)$.

Graph No. 1: Distribution of micro Pathogens

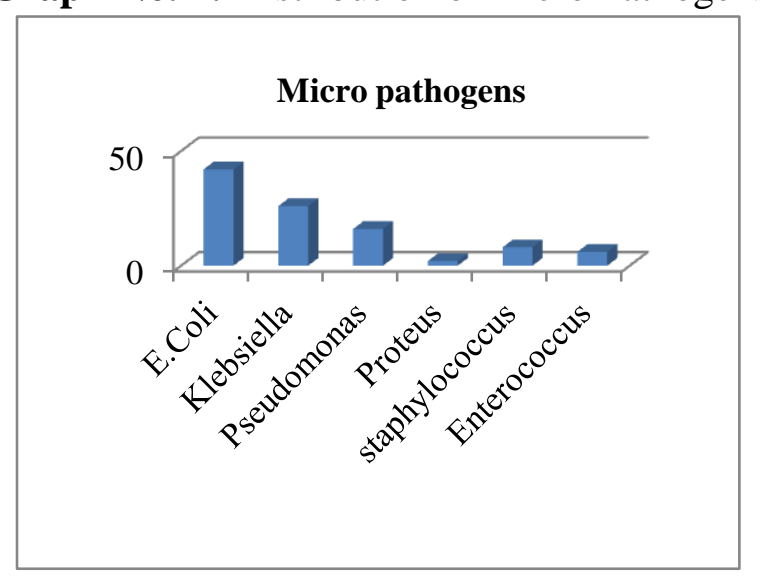




\section{Drug Sensitivity and Resistance}

As shown in Graph - 2, the drugs like ciprofloxacin, norfloxacin and ampicillin are found to be more resistant to microorganisms. The drugs like amikacin, gentamicin and cefoperazone sulbactam are found effective. In $6 \%$ of cases multiple drug resistance is also noticed.

Graph No. 2: Distribution of drug sensitivity and resistance

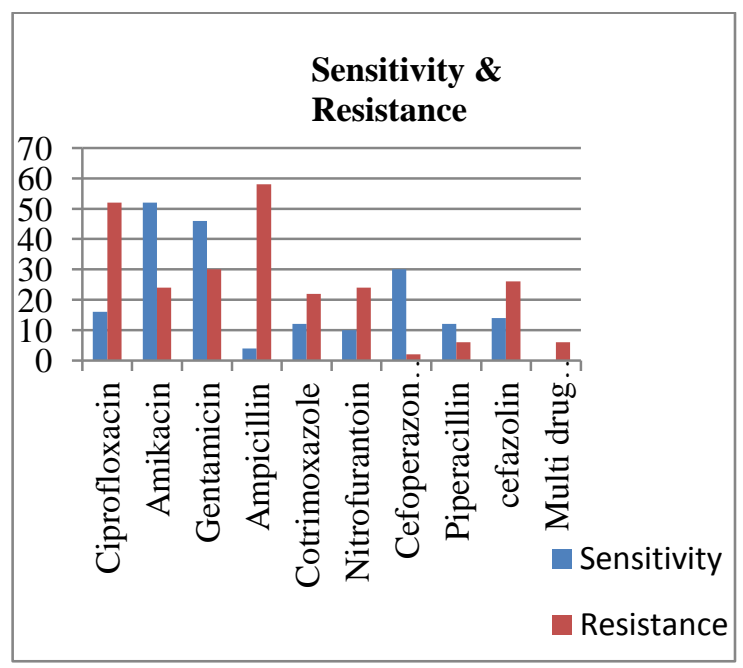

\section{DISCUSSION}

All most all patients with spinal cord injury suffer from urinary tract infection. It has been noted in the article by Biering-Sørensen F et.al that the UTIs is the leading cause for septicaemia, which increases morbidity in patients with spinal cord injury ${ }^{7}$. There are studies which indicate that, acute UTIs are mainly due to infection with E. coli in patients without catheters. Whereas in spinal cord injury patients with indwelling catheters, the prevalence of other organisms is much higher. In catheter associated infections, the Klebsiella, Pseudomonas and Proteus play an important role ${ }^{2}$. So we decided to conduct a study to determine the extent of prevalence of micro pathogens in urinary tract infections in catheterised spinal cord injury patient who are having UTIs. A cross sectional study was performed on 100 such patients, admitted in the Physical Medicine and Rehabilitation department. In this study, we found that $42 \%$ of true symptomatic UTIs are caused by E. coli, $26 \%$ by Klebsiella, $16 \%$ by
Pseudomonas, $2 \%$ Proteus, $8 \%$ by Staphylococcus aureus and $6 \%$ by entero coccus.

According to Juergen Pannek, treatment of asymptomatic urinary tract infections will increase the risk of developing multidrug resistance ${ }^{8}$. Hinkel et al.2 showed that over a 5-year period, there is a significant increase in the resistance of bacterial strains isolated in persons with SCI against commonly used antibiotics ${ }^{9}$. In another article by Waites et al., it has been highlighted that treatment of symptomatic UTI with ciprofloxacin in acute period will lead to the development of ciprofloxacin-resistant strains in more than $50 \%$ of the patients at follow-up ${ }^{10}$.

In our study, the culture reports are showing that there is resistance to drugs like ampicillin, ciprofloxacin, norfloxacin etc. In $6 \%$ of cases there is development of multidrug resistance. Intravenous antibiotics like cefoperazone sulbactum and piperacillin are found to be effective mainly against Pseudomonas aeruginosa.

According to Juergen Pannek, symptomatic UTIs should be treated with antibiotics based on the results of microbiologic testing if possible ${ }^{8}$. The pharmacological actions and adverse effects of the drug should also be considered before prescribing drugs, along with the microbiology report.

\section{CONCLUSIONS}

In the above study, it was found that, culture and sensitivity tests are important in the diagnosis and management of urinary tract infections in spinal cord injury patients.

Concluding,

1) In catheter associated urinary tract infections, apart from the usual gram negative bacilli E. coli, the Klebsiella and Pseudomonas showed significant prevalence.

2) Multi drug resistance are found to be increasing, may be due to recurrent and inappropriate antibiotic treatment.

3) Early detection of UTI and timely management will reduce morbidity and improves the quality of life. 
4) The duration of catheterisation should be minimal. Intermittent clean catheterisation should be started as early as possible, if not contraindicated.

\section{IMPLICATIONS}

In patients with spinal cord injury who are on indwelling catheter, urine culture and sensitivity tests should be done before starting the antibiotics.

\section{LIMITATIONS OF THE STUDY}

Study is done in one centre only. There is more scope for multicentre study.

\section{ACKNOWLEDGEMENT}

To all the patients and other members of the team involved in this study.

\section{REFERENCES}

1. Apurba Sankar Sastry, Sandhya Bhat K, Essentials of Medical Microbiology, First edition, chapter 29, Page 303.

2. Harrison's Internal Medicine $17^{\text {th }}$ edition, Chapter 282. Urinary Tract Infections, Pyelonephritis, and Prostatitis

3. Erickson RP, Merritt JL, Opitz JL, Ilstrup DM. Bacteriuria during follow-up in patients with spinal cord injury: I. Rates of bacteriuria in various bladder-emptying methods. Arch Phys Med Rehabil. 1982; 63:409-12

4. Ananthanarayan and Paniker's Text of Microbiology $9^{\text {th }}$ edition, Page 670- 672.

5. Burgdörfer H, Heidler H, Kutzenberger J, Madersbacher H, Palmtag H, Pannek J, et al. Manual neuro-urology and spinal cord lesion. 4th ed Köln, Germany: Farco; 2007

6. Everaert $\mathrm{K}$, Lumen $\mathrm{N}$, Kerckhaert $\mathrm{W}$, Willaert $\mathrm{P}$, van Driel M. Urinary tract infections in spinal cord injury: prevention and treatment guidelines. Acta Clin Belg 2009; 64(4):335-40.

7. Biering-Sørensen F1, Bagi P, Høiby N.Urinary tract infections in patients with spinal cord lesions: treatment and prevention. Drugs. 2001; 61(9):1275- 87.

8. Juergen Pannek. Treatment of urinary tract infection in persons with spinal cord injury: guidelines, evidence, and clinical practice. J Spinal Cord Med. 2011 Jan; 34(1): 11-15.

9. Hinkel A, Finke W, Bötel U, Gatermann SG, Pannek J. Increasing resistance against antibiotics in bacteria isolated from the lower urinary tract of an outpatient population of spinal cord injury patients.Urol Int 2004; 73(2): 143-8.

10. Waites KB, Canupp KC, Brookings ES, DeVivo MJ. Effect of oral Ciprofloxacin on bacterial flora of perineum, urethra, and lower urinary tract in men with spinal cord injury. J Spinal Cord Med 1999; 22(3):192-8. 